\title{
EMVA 1288 Camera characterisation and the influences of radiometric camera characteristics on geometric measurements
}

\author{
Maik Rosenberger, Chen Zhang, Pavel Votyakov, Marc Preißler, Rafael Celestre, Gunther Notni \\ IImenau University of Technology, Gustav Kirchhoff Platz 2, 98693 IImenau, Germany
}

\begin{abstract}
Over the past decades, a large number of imaging sensors based mostly on CCD or CMOS technology were developed. Datasheets provided by their developers are usually written on their own standards and no universal figure of merit can be drawn from them for comparison purposes. The EMVA 1288 is a standard aims to overcome this problem by setting parameters and experimental setup for radiometric characterisation of cameras. An implementation of an experimental setup and software environment for radiometric characterisation of imaging sensors following the guidelines of the EMVA 1288 is presented here. Using simulations, the influences and impact of several EMVA 1288 parameters on geometric measurements can be estimated. This paper also presents a signal model and image acquisition chain; measurements of radiometric characteristics of an image sensor; and sensor evaluation for geometric measurements, where the aforementioned influences on geometric measurements are discussed.
\end{abstract}

\section{Section: RESEARCH PAPER}

Keywords: camera characterisation; camera parameters; edge transition model; optical geometric measurements

Citation: Maik Rosenberger, Chen Zhang, Pavel Votyakov, Marc Preißler, Rafael Celestre, Gunther Notni, EMVA 1288 Camera characterisation and the influences of radiometric camera characteristics on geometric measurements, Acta IMEKO, vol. 5, no. 4, article 13, December 2016, identifier: IMEKO-ACTA05 (2016)-04-13

Section Editor: Paul Regtien, The Netherlands

Received March 22, 2016; In final form December 12, 2016; Published December 2016

Copyright: @ 2016 IMEKO. This is an open-access article distributed under the terms of the Creative Commons Attribution 3.0 License, which permits unrestricted use, distribution, and reproduction in any medium, provided the original author and source are credited

Funding: The presented work is the result of the research within the project ID2M which is situated at the Ilmenau University of Technology, Germany as part of the research support program InnoProfile, funded by the Federal Ministry of Education and Research (BMBF) Germany

Corresponding author: Maik Rosenberger, e-mail: maik.rosenberger@tu-ilmenau.de

\section{INTRODUCTION}

Over the last decades, plenty of different image sensors were developed by a huge variety of companies. Presently there is a strong trend to complementary metal oxide semiconductor based image sensors (CMOS) in comparison to the charge coupled device sensors (CCD) because of their high resolution and frame rate with lower power consumption [1] as well as their strong ability for integration of signal processing circuits down to pixel level [2]. On the other hand, the CMOS sensors are subjected to worse signal to noise ratio (SNR), as shown in [1]. Furthermore, the CMOS sensors have usually a fixed pattern noise (FPN) in the image due to their read out circuit [3], while the FPN in CCD sensors is mostly of a random nature.
Having this huge variety of sensor characteristics, there is a difficulty in the image sensor choice for practical applications, especially for geometric measurements in regard of the required high measurement stability and accuracy. First of all, the sensor comparison could be meaningfully conducted only under the precondition that the sensors are measured and characterised according to the same standard and with the same measuring instructions. However, the datasheets provided by the industrial camera manufacturers are usually written in their own standards and formats, so it is difficult to draw a comparison between different sensors using them. Opposite to this situation, the EMVA 1288 standard was released to define the measurement and characterisation methods of image sensors and the format of datasheets. 
Furthermore, there is a need for understanding the impact of image sensor parameters on image processing procedures. For high-precise optical 2D geometric measurements based on areas of interest consisting of $1 \mathrm{D}$ search lines, the fundament is the determination of edge point location with subpixel accuracy on each search line. Up until now, the theoretical investigations on edge detection (e.g. [4]-[6]) focus mainly on the performance comparison between different methods under a simple noise model that is not completely in agreement with the physical camera model and thus cannot present the real sensor characteristics. To estimate the influence of sensor parameters on real measurements, a systematic simulation model is needed.

The EMVA 1288 standard gives a mathematical description of the signal conversion procedure in the camera system which can be also used as the fundament of the simulation model. Moreover, the simulation should be designed considering real measurement conditions.

In this paper, a measurement setup for radiometric camera characterisation according to the EMVA 1288 standard is firstly described. Subsequently, a method based on a close-to-reality simulation model with the sensor parameters in the EMVA 1288 standard is proposed to evaluate image sensors in respect of geometric measurements in accordance with measurement metrology. Using this simulation method, the influences of several EMVA 1288 sensor parameters were estimated.

\section{SIGNAL MODEL AND IMAGE AQUISITION CHAIN}

In general, the task of image sensors is the transformation of electromagnetic radiation into analogue or digital signals. The fundament of this signal conversion process is the photoelectric effect. For image sensors in the ultraviolet-visible (UV-VIS) and near-infrared (NIR) range, mainly the inner photoelectric effect is utilized for the conversion of photons into electrical signals.

Figure 1 illustrates the signal conversion stages in the image acquisition. The photons interact with the silicon layer so that a number of electron-hole pairs are generated in dependence on the amount of photons. This effect results in an accumulation of elementary charges in a potential well. The collected charges are converted into voltage output in proportion to the amount of collected electrons in an active sensor area which are called pixels and can be assembled as a CCD or CMOS element. Finally, the voltage is quantized to discrete digital gray values with an analog-to-digital converter. The saturation of the potential well will lead to saturation of the gray value so that it won't no longer increase its value with further exposure.

Figure 1 lists uncertainty sources that are standardised in the EMVA 1288 camera model, which is illustrated in Figure 2. It begins with the generation of electrons during the exposure time. With the wavelength $\lambda$ dependent total quantum efficiency $\eta(\lambda)$, the average number of photons $\mu_{p}$ hitting the pixel area is converted into an electrical signal $\mu_{e}$ :

$\eta(\lambda) \cdot \mu_{p}=\mu_{\mathrm{e}}$

For (1), the effects generated by the fill-factor and the influences of the micro lenses mounted on the active sensor area will be included in the total quantum efficiency. The mean number of photons $\mu_{p}$ can be calculated using the knowledge about the pixel area $A$, exposure time $t_{\text {exp }}$ and the irradiance $E$ on the sensor surface according (2):

$\mu_{p}=\frac{A E t_{\text {exp }}}{h v}=\frac{A E t_{\text {exp }}}{h \frac{c}{\lambda}}$,

where $b$ is the Planck's constant and $c$ the speed of light.

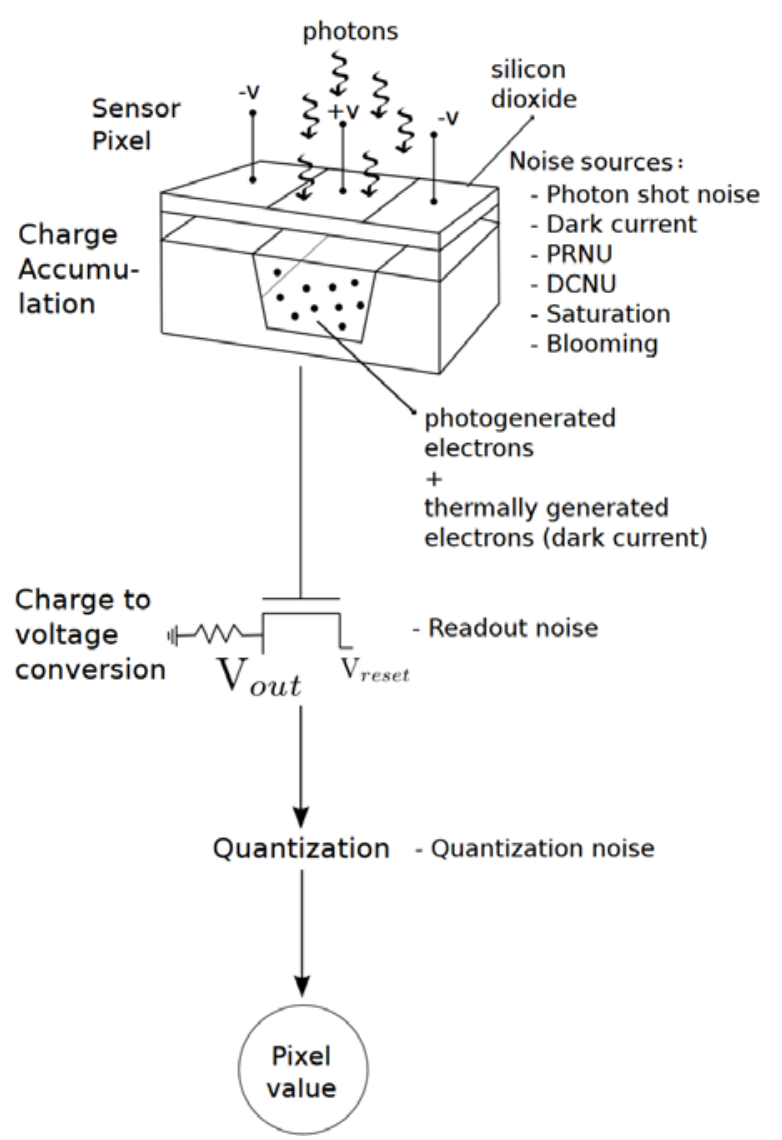

Figure 1: Simplified diagram of the image acquisition process and the principal noise sources [7].

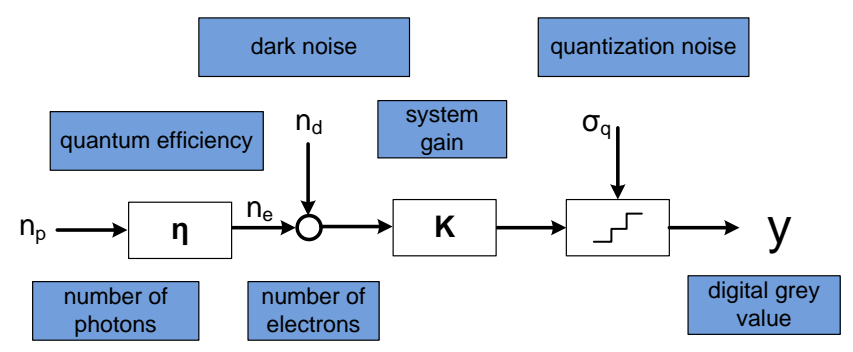

Figure 2: Schematic of the EMVA 1288 camera model [8].

For the calculation of $\mu_{p}$, a precise irradiance measurement is needed on the same place where the image sensor is under test. Besides, a minor number of electrons $\mu_{d}$ are also accumulated during the exposure time due to the sensor thermal effect as well as the uncertainty from the sensor read out and amplifier circuits. The dark and bright signals are summed and amplified and this behaviour is modelled using (3) with the introduction of the overall system gain factor $K$ :

$\mu_{y}=K\left(\mu_{d}+\mu_{e}\right)=\mu_{y . d a r k}+K \mu_{e}$.

In combination with (1) and (2), the mean gray value $\mu_{y}$ can be calculated as the sum of the mean value of the dark gray value and the product of overall system gain factor $K$ and expected number of electrons $\mu_{e}$ which is calculated from the number of photons:

$\mu_{y}=\mu_{y \cdot d a r k}+K \eta \frac{\lambda A}{h c} E t_{\text {exp }}$.

With this equation, the linearity of the sensor can be characterized using image gray values. 
The temporal fluctuation of the number of accumulated charge units, namely the shot noise, is subjected to a Poisson distribution [8], hence its variance can be determined with the mean value:

$\sigma_{e}^{2}=\mu_{e}=\left(\mu_{y}-\mu_{y . d a r k}\right) / K$.

With the introduction of the quantisation noise $\sigma_{q}$, a signal independent dark noise $\sigma_{d}$ as well as the photon shot noise $\sigma_{c}$ calculated with (5), the overall noise $\sigma_{y}$ measured with digital images can be characterized with (6):

$\sigma_{y}^{2}=\underbrace{K^{2} \sigma_{d}^{2}+\sigma_{q}^{2}}_{\text {offset }}+\underset{\text { slope }}{K}\left(\mu_{y}-\mu_{y . d a r k}\right)$.

As mentioned, the dark noise $\sigma_{d}$ consists of the thermal noise, which is Poisson distributed, since it is an accumulative process, and the read out and circuits noise that can be modelled with a Gaussian distribution [9].

From the radiation measurement, the dark noise $\sigma_{d}$ and the overall gain factor $K$ can then be measured from (4) to (6) with the photon transfer method [10]. For the determination of these parameters, a measurement setup was developed and is presented in Section 3.

\section{MEASUREMENT SETUP FOR THE DETERMINATION OF RADIOMETRIC IMAGE SENSOR PAMAMETERS}

\subsection{Measurement setup construction}

The requirements for the measurement setup to calculate the sensor parameters in the EMVA 1288 standard are given in the sections 6-9 of [8]. With the knowledge of those requirements, the measurement setup in Figure 3 was developed. The main criteria for the construction is to ensure the accordance with the pinhole camera model, which is realized with the $f$-number restriction:

$f_{\#}=\frac{d}{D}=8$.

With an $f$-number restriction of 8 and a given distance $d$ between the light source and the sensor plane, the free radiation diameter $D$ of the light source can be calculated. Another major point for this construction is the ideal wall surface behaviour inside the tube. Therefore, a special coating which ensures a minor reflection coefficient was used for the inner surface. Furthermore, a special camera socket was constructed for the reason to minimize the parasitic reflection inside the mounting.

\subsection{Software development}

The software for the EMVA 1288 measurement setup was developed using the Matlab environment. The software handles GigE-Vision cameras as well as pictures taken by the user with other systems. The complete standard was implemented in this measurement tool. Figure 4 illustrates the general procedure of the EMVA 1288 measurement. First of all, the irradiance which

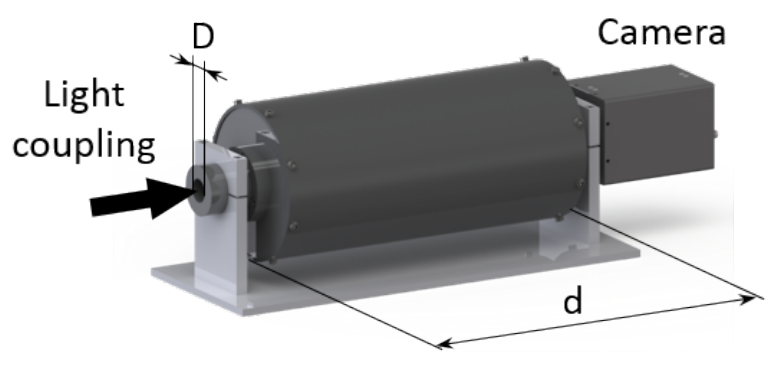

Figure 3: EMVA 1288 measurement setup.

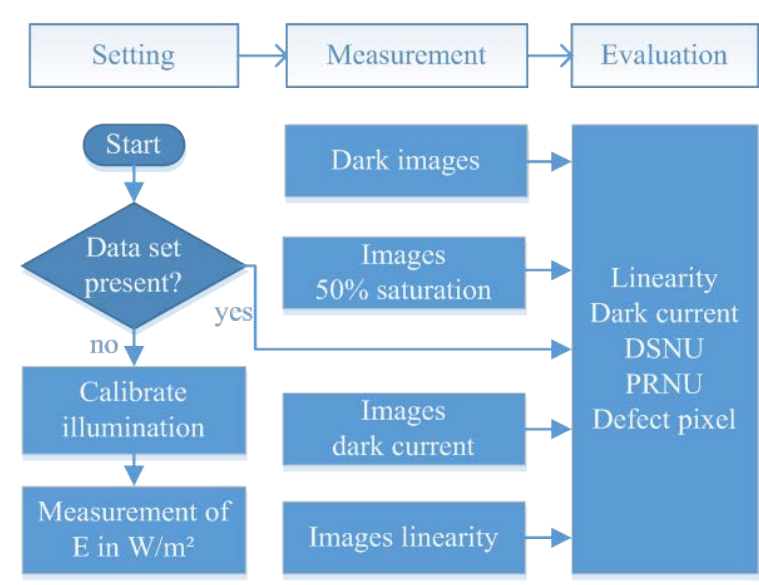

Figure 4: General procedure for data acquisition and evaluation separated in three stages.

will be then applied to the measurement position of the image sensor under test has to be measured with a radiometer. After the measurement of the radiation power, the test camera can be connected to the setup, afterwards the acquisition is started to measure the radiometric parameters and the non-uniformity of image sensor.

At the end of the camera characterisation, a standard compliant EMVA 1288 datasheet for the test camera can be generated automatically. This datasheet can then be used in the simulation program for the sensor evaluation with regard to its process capability in the high precision 2D optical geometric measurement based on search lines. The simulation program was also developed in Matlab with an interface for the import of the camera datasheet. Following the camera model in Figure 2, a model was developed for the simulation of the 1D edge detection process with subpixel accuracy, which is the crucial process in $2 \mathrm{D}$ measurements. With this simulation model and the measured sensor parameters, the sensor can be evaluated according to the measurement uncertainty resulting from the Monte Carlo simulation [11]. The simulation model and its implementation are described extensively in Section 5. Moreover, the sensor parameters can be freely scaled in the simulation program in order to estimate the influences of the individual sensor parameters on the measurement.

\section{MEASUREMENTS OF RADIOMETRIC IMAGE SENSOR CHARACTERISTICS}

The first measurements were taken with two CCD camera systems which differ in pixel size and quantum efficiency. The special requirement for this measurement is the equal set of the exposure time levels, so that a comparison between the cameras becomes possible.

In Figure 5, the different saturation points as well as the difference in the sensitivity coefficient between both cameras are easy to recognise. The saturation point of the CCD sensor ICX 415AL with higher sensitivity is reached at 34000 photons per pixel, which is significantly lower than ICX 424.

\section{SENSOR EVALUATON FOR GEOMETRIC MEASUREMENTS BASED ON EMVA 1288 SENSOR PARAMETERS}

\subsection{Simulation model}

In the majority of previous works about the simulation of $1 \mathrm{D}$ edge detection, e.g. [4], [5] and [6], it is simply assumed that 


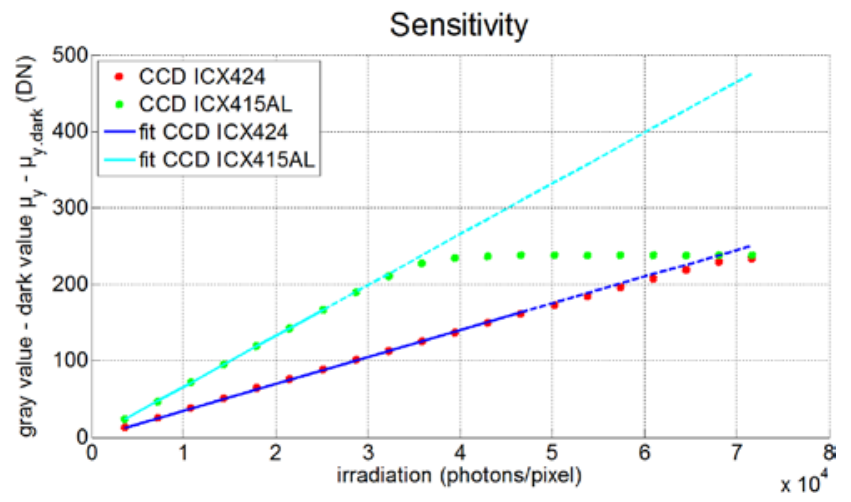

Figure 5: Sensitivity measurements at different camera systems.

the noise follows a Gaussian distribution. But this assumption could not conform to the real radiometric sensor characteristic according to the camera model in EMVA 1288. In this work, a simulation model based on this camera model was developed with consideration of all noise sources.

For the 1D edge detection, a linear image must be generated first. This is considered as a cut from the $2 \mathrm{D}$ image. The simulation aims for the evaluation of measurement capability of the whole sensor area, hence the sensor non-uniformity which is characterised with $\mathrm{DSNU}_{1288}$ and $\mathrm{PRNU}_{1288}$ is also modelled as random parameters in the $1 \mathrm{D}$ image simulation.

Figure 6 illustrates the process to simulate the measurement uncertainty of edge detection with given radiometric sensor parameters. At the first step, a spatial distribution of irradiation in metric units is given as the light signal on the image sensor.

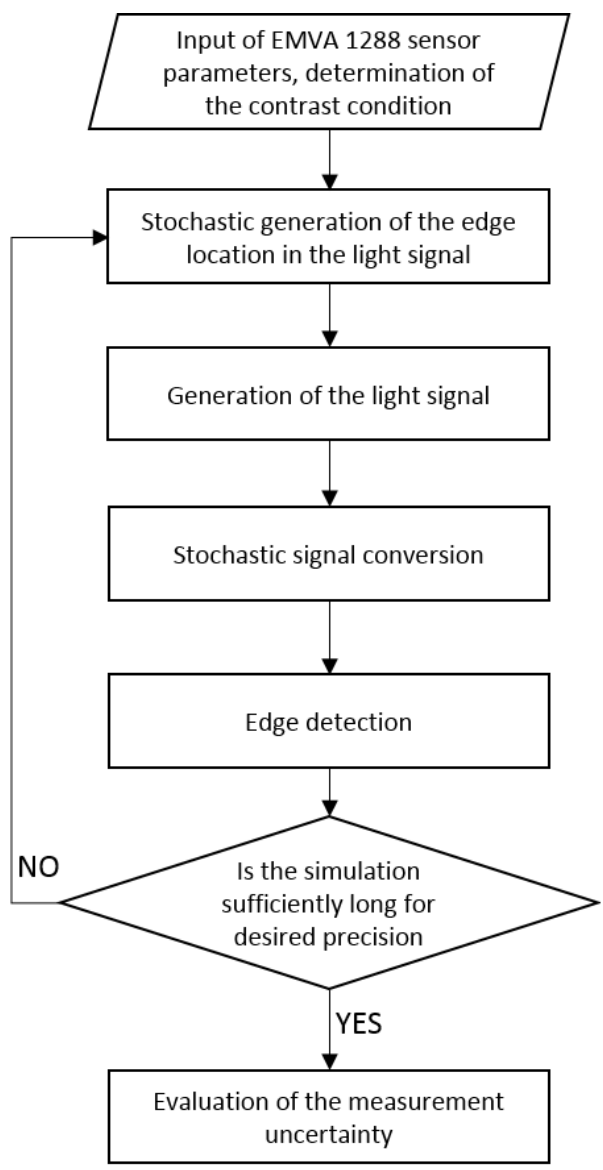

Figure 6: Simulation of measurement uncertainty of edge detection.
Since it is proved in [12] that the edge detection error is strongly dependent on the difference between the edge location and the center of the corresponding sensor pixel, a moving edge is used whose subpixel-part is generated randomly in the value range between 0 and 1 using a uniform distribution function.

Considering the blurring effect due to the imaging optics, the blurred edge transition model in [13] under the assumption of a Gaussian point spread function (PSF) is used. This edge model is showed in Figure 7 and formulated in (8):

$I(x)=\frac{k}{2}\left(\operatorname{erf}\left(\frac{x-l}{\sqrt{2} \sigma}\right)+1\right)+h$,

where $l$ is the edge location, $b$ the intensity offset, $k$ the edge contrast, and $\sigma$ the standard deviation of the Gaussian PSF.

This signal is then converted into a discrete distribution of gray values along a defined number of pixels in the following step. At first, the number of photons in each pixel is calculated by integrating (8) over the pixel grid $L$. The number of photons in the $n$-th pixel is given by (9):

$\mu_{p}(n)=\int_{(n-1) \cdot L}^{n \cdot L} 50.34 \cdot t_{\text {exp }} \cdot \lambda \cdot I(x)$.

In this equation, the light wavelength and the exposure time are set constant and their values are $550 \mathrm{~nm}$ and $1 \mathrm{~ms}$, respectively.

From the number of photons, the gray value of each pixel is simulated according to the system model in Figure 2. Upon the assumption that the linearity error characteristic is unchanged over the sensor area, the quantum efficiency $\eta$ is pixel-wisely adjusted to $\eta_{l}$ according to the number of irradiated photons and the linearity error curve. In the next step, the spatial nonuniformity of photon response is simulated. According to [14], a Gaussian random function with $\eta_{l}$ as mean and $\mathrm{PRNU}_{1288}$ as variance is used to generate the final quantum efficiency $\eta_{l, p}$ for each pixel. Then, the bright signal $\mu_{e}$ with photon shot noise is generated using a random Poisson distribution function with mean value $\eta_{l, p} \cdot \mu_{p}$.

In the simulation of the dark signal which is considered as thermally induced electrons, a basic noise signal $\mu_{d, g}$ is firstly generated from a random Poisson distribution function whose mean value is the square of the dark noise value. To simplify the spatial non-uniformity, only the spatial variance is taken into consideration, in disregard of the periodic variations. As in the non-uniformity simulation at the bright signal, a Gaussian random function with $\mu_{d, g}$ as mean and DSNU 1288 as variance is used to generate the final dark signal $\mu_{d}$.

The bright and dark signal are combined with each other, then multiplied with the gain factor $K$ and quantized to gray values. The edge location is detected with an adaptive threshold

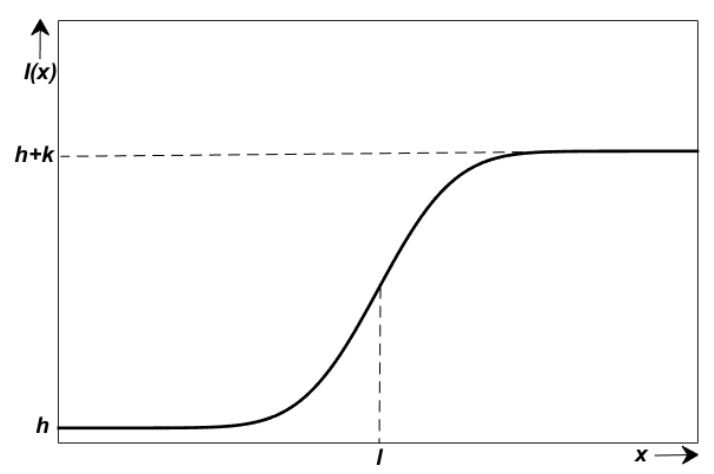

Figure 7: Edge transition using specific blurred edge model. 
that is estimated using the histogram based method in [15]. To achieve the subpixel accuracy, a cubic interpolation is used in the edge transition area and the subpixel edge point is defined as the intersection of the threshold and the fitted function.

The complete procedure is repeated according to the Monte Carlo method [11]. The deviation of the detected edge from the defined edge location in (8) is used as outcome in each iteration. Under the assumption that the distribution of the edge detection error is subjected to a symmetric distribution function, the outcomes are evaluated with the quantile method for symmetric distributions [15] to determine the measurement uncertainty (MU), which is defined as half of the interval having a $95 \%$ level of confidence [16].

\subsection{Model implementation}

In the model implementation the parameters in (8) must be determined first. The $\sigma$ value is to be set according to the characteristic of the optics which will be used in the real measurements. The allowed high level $b+k$ in the irradiation distribution (Figure 7) is determined based on the gray value simulation at a single pixel using the sensor data und the camera model. In this process, the irradiation with which the possibility of pixel saturation equals to $95 \%$ is determined by simulation and used as the high level in the light signal. Based on the high level $b+k$, the edge transition contrast in (8) can be flexibly set by varying the offset $b$ to simulate different measurement conditions in the practice.

An important parameter in the Monte Carlo method is the number of iterations $n$. This number should be as small as possible to save simulation time but ensure a stable simulation result at the same time. The ratio of the standard deviation $S_{U}$ of the simulated measurement uncertainty to its mean value $\bar{U}$ is utilized to examine the simulation stability. Figure 8 illustrates the dependency of simulation stability on the iteration number. It is shown that this ratio remains nearly constant at $0.13 \%$ from $n=50000$, hence all the simulations are performed with 50000 iterations.

The simulation result using sensor parameters of the CCD sensor "ICX445" is shown in Figure 9. It can be shown that the deviation of the detected edge location from its target value agrees very well with a symmetrical distribution. Hence, the use of the quantile method can be validated.

\subsection{Analysis of the influence of sensor data on the uncertainty of measurement}

Based on this simulation model and the real characteristics of the CCD sensor "ICX445", the influences of linearity error, dark noise, $\mathrm{DSNU}_{1288}$ and $\mathrm{PRNU}_{1288}$ on the measurement uncertainty were estimated by a gradual raise of individual parameters, while the other system parameters remained

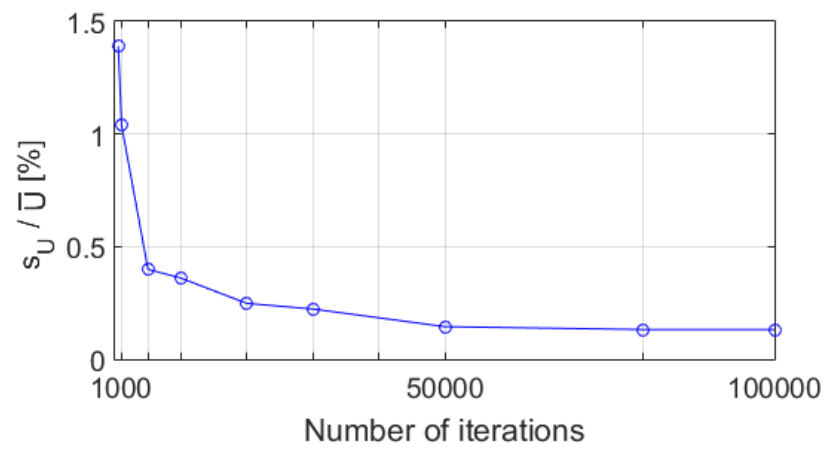

Figure 8: Stability of simulation in dependency on the number of iterations.

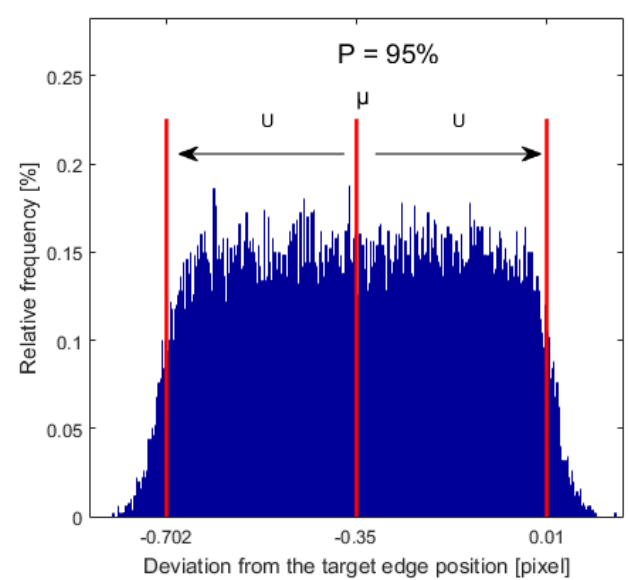

Figure 9: Simulation result with sensor parameters of CCD-sensor "ICX445".

unchanged. The $\sigma$ value in (8) is set to 5 , with which the edge transition form nears the real characteristics delivered by optical systems. The tests were at first performed with $100 \%$ contrast, in which the light signal covers the full sensor dynamic range. Afterwards, the contrast dependence of the influences of these sensor parameters was investigated with gradually reduced contrast by raising the low level in the light signal. The results of the investigations are represented in Figure 10 to Figure 13. In the datasheet the dark noise and $\mathrm{DSNU}_{1288}$ are given with the absolute unit [e-], which cannot directly indicate their relation to the 8 -bit digitalized image signal. Therefore, these parameters are represented with the ratio between them and the saturation capacity of the image sensor. Moreover, a change of

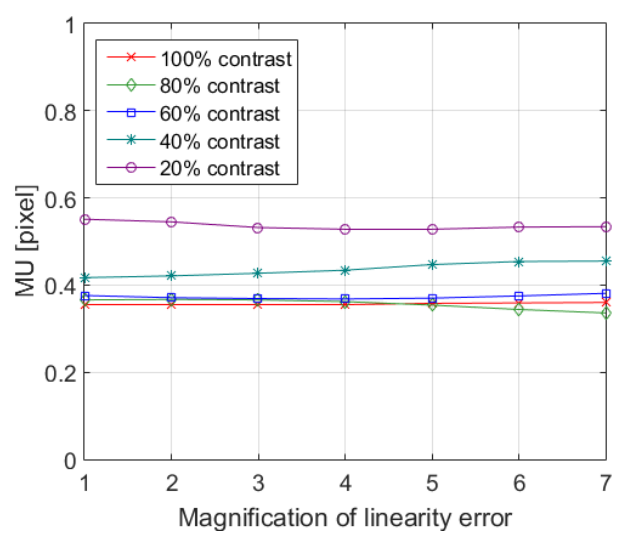

Figure 10: Measurement uncertainty with magnification of linearity error.

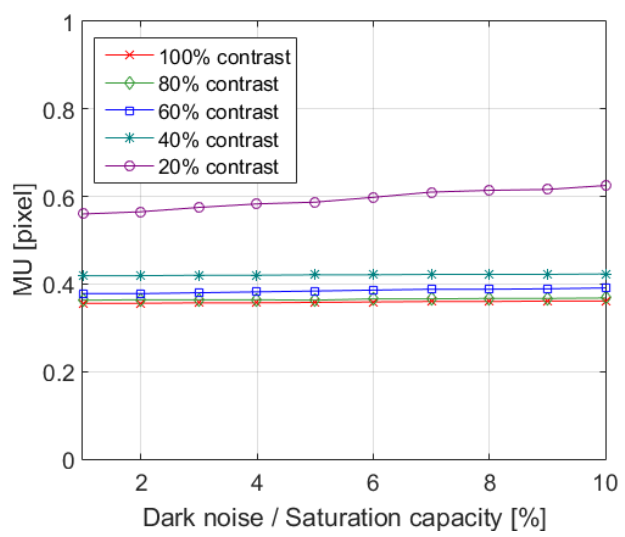

Figure 11: Measurement uncertainty with magnification of dark noise. 


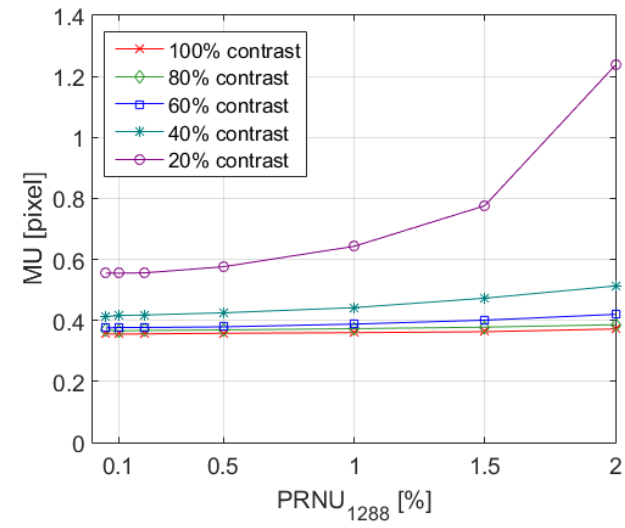

Figure 12: Measurement uncertainty with magnification of $\mathrm{PRNU}_{1288}$.

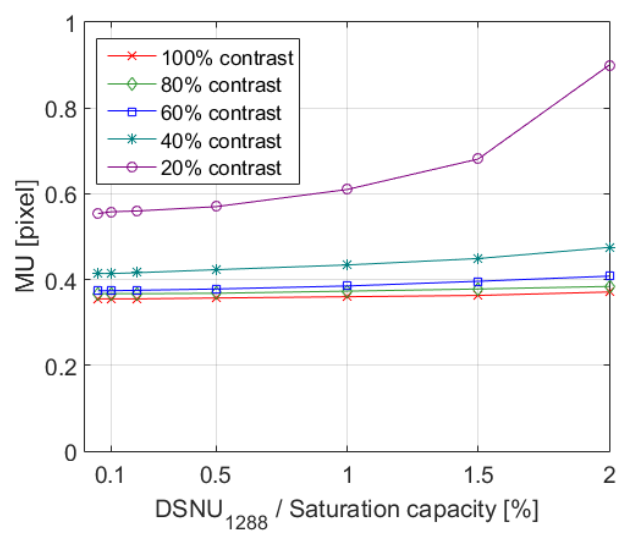

Figure 13: Measurement uncertainty with magnification of $\mathrm{DSNU}_{1288}$.

systematic measurement deviation is expected with the magnification of linearity error, hence the variation of the expected value of the absolute measurement deviation were also observed in this case.

With the full contrast that refers to the optimal illumination condition, the magnification of linearity error and dark noise cause hardly any significant change of measurement uncertainty (smaller than 0.005 pixel), though the magnification of the linearity error causes a systematic shift of the detected edge location, as shown in Figure 14.

Likewise, the magnification of $\mathrm{PRNU}_{1288}$ and $\mathrm{DSNU}_{1288}$ up to $0.5 \%$ brings hardly any changes. With a further magnification of these parameters, a minor rise of measurement uncertainty could be observed. As these parameters expand to 2 $\%$, the uncertainty rises by ca. 0.015 pixel. As a summary, the test results are robust in resistance of a certain deterioration of sensor parameters under optimal contrast conditions, because an adequate dynamic range in the converted gray value image is fundamentally secured in this illumination situation.

As the contrast decreases to $60 \%$, all the measurement uncertainty curves move slowly upwards with only one exception in Figure 10. With a further decrease of contrast, this movement becomes significant. The reason for the growth of measurement uncertainty is that the decrease of contrast raises the ratio between the uncertainty of the individual gray values in the signal and its dynamic range by reducing the resulting value.

On decreased contrast levels, the magnification of linearity error can cause an irregular change of uncertainty, whereby the value change is lower than 0.04 pixel. The characteristic of the

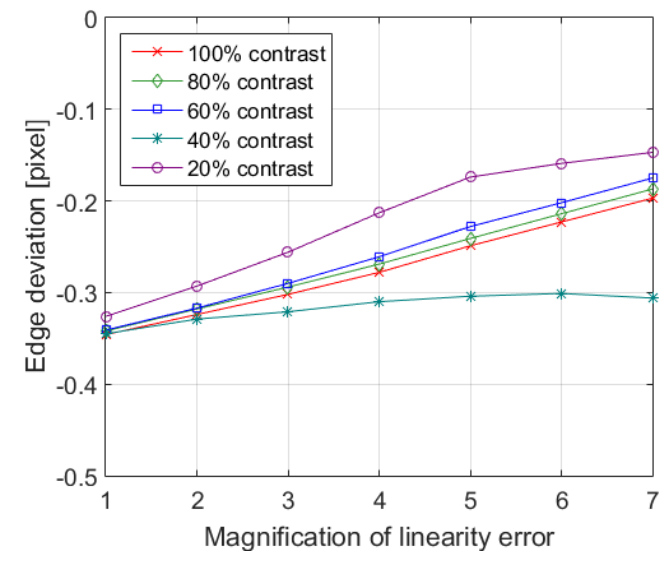

Figure 14: Measurement deviation with magnification of linearity error.

systematic measurement deviation remains nearly the same in $80 \%$ and $60 \%$ contrast, but shows a greatly irregular changes in $40 \%$ and $20 \%$ contrast, as shown in Figure 14.

The reason is speculated to be that the linearity error of the sensor is not uniformly distributed over the irradiation levels, as shown in Figure 15, so the in $40 \%$ and $20 \%$ contrast used sensor ranges have different linearity error characteristics.

With the contrast decrease down to $40 \%$, the measurement uncertainty remains still stable against the magnification of dark noise. A clear relationship between dark noise and uncertainty could only be observed under the $20 \%$ contrast. In this case, the measurement uncertainty rises by 0.063 Pixel, as the dark noise expands to $10 \%$ of the saturation capacity.

A significant interaction between contrast level and sensor parameters can be observed at $\mathrm{PRNU}_{1288}$ and $\mathrm{DSNU}_{1288}$. On the $40 \%$ contrast an obvious rise of measurement uncertainty from the turning points at $0.2 \%$ in both curves can be already observed, whereby the uncertainty rises by 0.1 pixel with the magnification of $\mathrm{PRNU}_{1288}$ to $2 \%$ and by 0.06 pixel with the magnification of $\mathrm{DSNU}_{1288}$. At the $20 \%$ contrast, the increase of measurement uncertainty becomes more significantly. It rises by 0.68 pixel with the magnification of $\mathrm{PRNU}_{1288}$ and by 0.346 pixel with the magnification of $\mathrm{DSNU}_{1288}$.

From the results above, it can be seen that for low light applications and in the case of weak reflective object surfaces the sensor parameters $\mathrm{PRNU}_{1288}$ and $\mathrm{DSNU}_{1288}$ have a strong influence on the measurement uncertainty in edge detection and should be considered primarily in sensor selection.

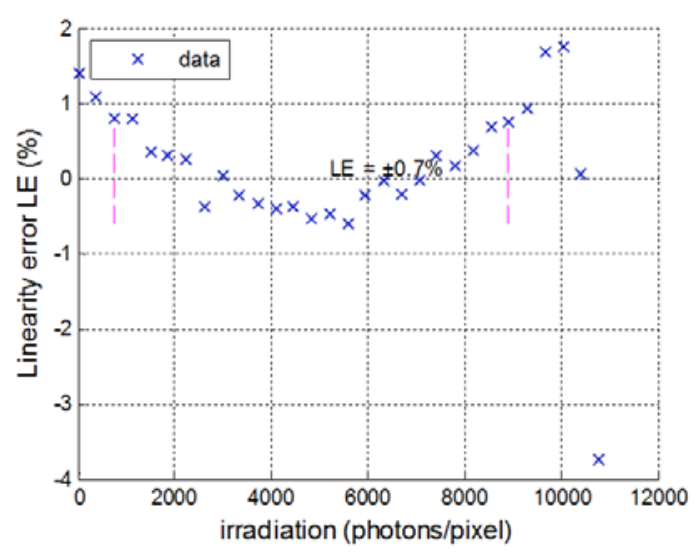

Figure 15: Linearity error curve of the CCD sensor "ICX445". 


\section{DISCUSSION OF RESULTS}

The magnification of the linearity error curve has generally a relatively weak influence on the measurement uncertainty but a significant influence on the systematic measurement deviation. The reason for it is that the measurement uncertainty results primarily from the uncertainty of the discrete gray values in the edge transition area, whereas the linearity error causes form distortion of the edge transition signal, which has a direct effect on the systematic measurement deviation. The irregularity of the influence on measurement uncertainty lies in the complex relationship between the signal form and the measurement uncertainty.

A high dark noise reduces the reachable signal-to-noise ratio of the sensor and thus the dynamic range of signal which correlates with the measurement uncertainty. This effect becomes significant only when the contrast in the light signal is reduced to $20 \%$ and the dark noise is extremely high.

The parameters $\mathrm{PRNU}_{1288}$ and $\mathrm{DSNU}_{1288}$, which directly refer to the uncertainty of the individual gray values, contribute the most to the measurement uncertainty. The reason for the difference of the curve characteristics shown in Figure 12 and Figure 13 is that both these parameters represent two different sources of uncertainty. PRNU 1288 refers to the uncertainty of the photon response so that the bright pixels have a higher uncertainty, while the by $\mathrm{DSNU}_{1288}$ characterized dark noise uncertainty is the same to all pixels.

\section{CONCLUSIONS}

The presented summary shows an abstract of the possibility to characterize image sensors using the standard EMVA 1288 and to evaluate image sensors for geometric measurements using the Monte Carlo method in which the imaging process is simulated using the system model in the EMVA 1288 standard. With the simulation program, the influences of several essential parameters of image sensor on geometric measurements were investigated.

\section{REFERENCES}

[1] H. T. Beier and B. L. Ibey, "Experimental Comparison of the High-Speed Imaging Performance of an EM-CCD and sCMOS
Camera in a Dynamic Live-Cell Imaging Test Case", PLoS ONE 9(1):e84614, 2014.

[2] A. E. Gamal and H. Eltoukhy, "CMOS Image Sensors", IEEE Circuits and Devices Magazine, Vol. 21, Issue: 3, 2005.

[3] S. Mohammadnejad, S. Roshani and M. N. Sarvi, "Fixed Pattern Noise Reduction Method in CCD Sensors for LEO Satellite Applications", Proceedings of 11th International Conference on Telecommunications, 2011.

[4] E. P. Lyvers, O. R. Mitchell, M. L. Akey, and A. P. Reevs, "Subpixel measurements using a moment-based edge operator", IEEE Transactions on Pattern Analysis Machine Intelligence, vol.11, no.12, pp.1293-1309, 1989.

[5] F. Bouchara, M. Bertrand, S. Ramdani and M. Haydar, "Subpixel Edge Fitting Using B-Spline", Proceedings of the $3^{\text {rd }}$ International Conference on Computer Vision, pp. 353-364, 2007.

[6] M. Hagara and O. Ondráček, "Moving edge detection with sub pixel accuracy in 1-D images", Proceedings of $26^{\text {th }}$ International Conference Radioelektronika, 2016.

[7] C. Aguerrebere, J. Delon, Y. Gousseau and P. Musé, "Study of the digital camera acquisition process and statistical modeling of the sensor raw data", 2013. <hal-00733538v4>

[8] European Machine Vision Association, EMVA Standard 1288 Standard for Characterisation of Image Sensors and Cameras, Release 3.0, 2010.

[9] R, Mancini, Op Amps for everyone, Texas Instruments, 2002.

[10] J. R. Janesick, "CCD characterisation using the photon transfer technique", Solid State Imaging Arrays, Vol. 570 of SPIE Proc., pp. 7-19, 1985.

[11] D. P. Kroese, T. Taimre, Z. I. Botev, Handbook of Monte Carlo Methods, ISBN 978-0470177938, Wiley; 1. Edition, 2015.

[12] M. Hagara and O. Ondráček, "Comparison of methods for edge detection with sub-pixel accuracy in 1-D images", Proceedings of 3. Mediterranean Conference on Embedded Computing (MECO), pp. 124-127, 2014.

[13] M. Hagara, "Edge Detection with Sub-pixel Accuracy Based on Approximation of Edge with Erf Function", Radio Engineering, Vol. 20, Issue 2, 2011.

[14] M. Konnik and J. Welsh, "High-level numerical simulations of noise in CCD and CMOS photosensors: review and tutorial", eprint arXiv:1412.4031, 2014.

[15] K. Weißensee, "Beitrag zur automatisierbaren Messunsicherheitsentwicklung in der Präzisionskoordinatenmesstechnik mit Bildsensoren", $\mathrm{PhD}$ Thesis, Ilmenau University of Technology, 2011.

[16] Joint Committee for Guides in Metrology, Guide to the expression of uncertainty in measurement, 1. Edition, 2008. 